TRANSACTIONS OF THE

AMERICAN MATHEMATICAL SOCIETY

Volume 353, Number 6, Pages 2513-2530

S 0002-9947(01)02586-7

Article electronically published on February 15, 2001

\title{
TOPOLOGICAL HORSESHOES
}

\author{
JUDY KENNEDY AND JAMES A. YORKE
}

\begin{abstract}
When does a continuous map have chaotic dynamics in a set $Q$ ? More specifically, when does it factor over a shift on $M$ symbols? This paper is an attempt to clarify some of the issues when there is no hyperbolicity assumed. We find that the key is to define a "crossing number" for that set $Q$. If that number is $M$ and $M>1$, then $Q$ contains a compact invariant set which factors over a shift on $M$ symbols.
\end{abstract}

\section{INTRODUCTION}

The most basic and striking results in chaotic dynamics are perhaps the results about Smale horseshoes $[\mathbf{S}$. The theory concerns a set $Q$ (usually diffeomorphic to a rectangle) in a two-dimensional manifold $M$ and a diffeomorphism $f: Q \rightarrow M$. Using only hypotheses on the first iterate of $f$ on $Q$, one obtains conclusions about all iterates of $f$. In particular, Smale concludes that there is a compact invariant set $Q_{I}$ in $Q$ which is homeomorphic to a shift on $M$ symbols, where $M$ is often taken to be 2 . In this paper we will call $M$ the "crossing number" and our definition of this number for more general maps and sets $Q$ is central to our analysis of the chaos present in our dynamical systems.

Results such as Smale's are useful for numerical studies of systems with positive entropy because there are unavoidable errors in the computation of $f(Q)$ that can be made small by careful computation, but the errors in the computation of $f^{n}(Q)$ increase exponentially fast as $n$ increases, due to the positive entropy. Hence hypotheses on $f(Q)$ are easier to verify directly than hypotheses on $f^{n}(Q)$.

However, Smale's assumption of hyperbolicity of $f$ on $Q$ is in practice difficult to verify numerically. When the hyperbolicity assumption is discarded, the dynamics remain just as rich as before in that there is a compact invariant set $Q_{I}$ in $Q$ that factors over a shift on $M$ symbols, although the map need not be one-to-one. K. Burns and H. Weiss [BW] call such maps "geometric horseshoes," and the term "topological horseshoes" is also often used. (In their paper aimed at discussing situations where stable and unstable manifolds cross but do not cross necessarily transversally, they concluded that there is a geometric horseshoe for some iterate of the map.) K. Mischaikov et al. [CKM, CM, [MM] and A. Szymczak [S] investigate such situations using simplicial decompositions that can be analyzed rigorously numerically, showing for example that there is a subshift of finite type in the Lorenz

Received by the editors March 16, 1998 and, in revised form, December 21, 1998.

1991 Mathematics Subject Classification. Primary 58F12, 54F20; Secondary 54F50, 58F20.

Key words and phrases. Topological horseshoe, geometric horseshoe, chaos, shift dynamics, connection, preconnection, crossing number, expander, symbol set.

This research was supported by the National Science Foundation, Division of Mathematical Sciences. 
system. Example 3(c) in Section B of this paper is one in which shift dynamics is predicted by our results but to which the results of Szymczak and Mischaikov et al do not apply (because no isolating neighborhood exists).

Our goal here is to formulate the idea of the crossing number $M$ and to show that with this formulation and a few other hypotheses, it follows that there exists an invariant set $Q_{I}$ in $Q$ that factors over an $M$-shift. (The $M$-shift is one-sided if $f$ is only assumed to be continuous, or two-sided if $f$ is a homeomorphism.) In particular, we make no assumption that there exists a Markov partition on $Q$, nor do we assume that the continuous map $f$ is differentiable at any point.

If $f$ is continuous, we say $\mathcal{S}:=\left\{S_{i}\right\}_{i=1}^{M}$ is a set of symbol sets if for each sequence $\left(j_{i}\right)_{i=0}^{\infty}$ of integers in $\{1,2, \ldots, M\}$, there is a trajectory $\left(x_{i}\right)$ (where $x_{i+1}=f\left(x_{i}\right)$ for all $i$ ) such that $x_{i}$ is in $S_{j_{i}}$. If $f$ is a homeomorphism, we say $\mathcal{S}=\left\{S_{i}\right\}_{i=1}^{M}$ is a set of symbol sets if for each sequence $\left(j_{i}\right)_{i=-\infty}^{\infty}$ of integers in $\{1,2, \ldots, M\}$, there is a trajectory $\left(x_{i}\right)$ such that $x_{i}$ is in $S_{j_{i}}$. If the $S_{i}$ are disjoint, it follows that the invariant set $S$ contained in $\bigcup S_{i}$ can be mapped continuously onto a shift on $M$ symbols. Each $S_{i}$ in $\mathcal{S}$ is, naturally enough, a symbol set.

Establishing the existence and dynamics of $Q_{I}$ can be broken into two problems: first the construction of the symbol sets $S_{i}$, and second showing that they yield the required dynamics. The set of symbol sets $S_{i}$ we construct are mutually disjoint. The construction is carried out in two lemmas. The second step, where they yield the desired dynamics, is carried out via a lemma that we call the "Chaos Lemma". It was formulated with Sahin Koçak to be able to handle a variety of problems which will be investigated in a a separate paper [KKY]. We only sketch the proof in this paper. We also give a number of examples showing how our results can be applied. In the last section, we show that our results can be applied in a still wider variety of situations, by proving two lemmas that demonstrate that the horseshoe hypotheses $\boldsymbol{\Omega}$ (listed below) hold in the presence of a weaker set of hypotheses.

Throughout this paper (except in the Chaos Lemma, and the last section, where we relax our hypotheses further) we make the following five assumptions:

$\boldsymbol{\Omega}_{X}: X$ is a separable metric space.

$\boldsymbol{\Omega}_{Q}: Q \subset X$ is locally connected and compact.

$\boldsymbol{\Omega}_{f}$ : The map $f: Q \rightarrow X$ is continuous.

$\boldsymbol{\Omega}_{E}$ The set $e n d_{0} \subset Q$ and $e n d_{1} \subset Q$ are disjoint and compact, and each component of $Q$ intersects both $e n d_{0}$ and $e n d_{1}$.

$\boldsymbol{\Omega}_{M}: Q$ has crossing number (defined below) $M \geq 2$.

We refer to the five hypotheses $\boldsymbol{\Omega}_{X}, \boldsymbol{\Omega}_{Q}, \boldsymbol{\Omega}_{f}, \boldsymbol{\Omega}_{E}$, and $\boldsymbol{\Omega}_{M}$ collectively as the horseshoe hypotheses $\Omega$. A connection $\Gamma$ is a compact connected subset of $Q$ that intersects both end $d_{0}$ and $e n d_{1}$. (We use the term 'connection' because one might say $\Gamma$ connects $e n d_{0}$ to $e n d_{1}$.) Note that by hypothesis $\Omega_{E}$, each component of $Q$ is a connection. An example of a connection is a path in $Q$ intersecting both $e n d_{0}$ and end $d_{1}$. A preconnection $\gamma$ is a compact connected subset of $Q$ for which $f(\gamma)$ is a connection. We define the crossing number $M$ to be the largest number such that every connection contains at least $M$ mutually disjoint preconnections. We say a set $S$ is invariant if $f(S)=S$.

Our main result is the following theorem, which follows from the Chaos Lemma and Lemma 12 
Theorem 1. Assume the horseshoe hypotheses $\boldsymbol{\Omega}$. Then there is a closed invariant set $Q_{I} \subset Q$ for which $f \mid Q_{I}$ is semiconjugate to a one-sided $M$-shift. (If $f$ is a homeomorphism, then $f \mid Q_{I}$ is also semiconjugate to a two-sided $M$-shift.)

By assumption $\Omega_{M}$, each connection contains at least $M$ mutually disjoint preconnections. We prove the theorem by constructing $M$ compact mutually disjoint sets $S_{1}, S_{2}, \ldots, S_{M}$ such that each connection $\Gamma$ contains a preconnection in $S_{i} \cap \Gamma$ for each $i=1, \ldots, M$. This construction is quite difficult despite the fact that in most examples it is quite easy to find $S_{1}, \ldots, S_{M}$. Once the sets $S_{1}, \ldots, S_{M}$ are shown to exist, we can apply the following lemma by letting $E$ be the set of all connections. As stated below however, the lemma is more general than needed in that the sets in $E$ need not be connected.

Lemma 2. The Chaos Lemma. Assume $\boldsymbol{\Omega}_{X}, \boldsymbol{\Omega}_{Q}$, and $\boldsymbol{\Omega}_{f}$. Let $M$ be an integer greater than or equal to 2 . Let $E$ be a nonempty set of nonempty compact sets such that for each $\gamma \in E$ there exists a compact $\gamma_{i} \subset \gamma \cap S_{i}$ for which $f\left(\gamma_{i}\right) \in E$. Let $Q_{I}$ be the largest invariant set in $\bigcup S_{i}$. Then $f \mid Q_{I}$ is semiconjugate to a onesided shift on $M$ symbols. If $f \mid Q_{I}$ is one-to-one on $Q_{I}$, the restricted map $f \mid Q_{I}$ is semiconjugate to a two-sided shift on $M$ symbols.

\section{Notation, DEFinitions, BACKGROUND}

We use the term 'space' to mean 'separable metric space'. The word 'component' means 'connected component'. In the results that follow, we consider the topology on the closed subset $Q$ of the space $X$ only. Thus, 'open' means 'open relative to $Q$ ' and 'closed' means 'closed relative to $Q$ '. The sets in which we are interested are all contained in $Q$, and this means the restriction to $Q$ avoids our having to add repeatedly ' $\cap Q$ ' to the sets discussed. If $A$ is a subset of $Q$, then we use the notation $A^{\circ}, \bar{A}$, and $\partial A$ to denote the interior, closure, and boundary of $A$ in $Q$, respectively. A continuum is a compact, connected metric space. In this paper we allow a continuum to consist of just one point or to be the empty set. A subset of a continuum which is itself a continuum is a subcontinuum. If $A$ and $B$ are nonempty closed subsets of a space $X$, and $K$ is a continuum in $X$ which intersects both $A$ and $B$, then $K$ is a continuum irreducible from $A$ to $B$ if no proper subcontinuum of $K$ intersects both $A$ and $B$. If $A$ and $B$ are nonempty closed subsets of a space $X$, and $K$ is a continuum in $X$ which intersects both $A$ and $B$, then $K$ contains a continuum $K^{\prime}$ irreducible from $A$ to $B$. A chain is a finite collection $C=\left\{C_{0}, C_{1}, \ldots, C_{n}\right\}$ of sets listed so that $C_{i} \cap C_{j} \neq \varnothing$ if and only if $|i-j| \leq 1$. The members $C_{i}$ of the chain $C$ are called links. A tiling chain is a chain $C=\left\{C_{0}, C_{1}, \ldots, C_{n}\right\}$ each member of which is a closed set with nonempty interior and which has the property that for each $i \neq j, C_{i} \cap C_{j}=\partial C_{i} \cap \partial C_{j}$. For $0 \leq i<n$, we say that $\partial C_{i} \cap C_{i+1}$ is the right boundary of the link $C_{i}$, and for $0 \leq i \leq n$, we say that $\partial C_{i} \cap C_{i-1}$ is the left boundary of the link $C_{i}$.

Note that in the discussion of the horseshoe hypotheses in the previous section, the sets $e n d_{0}$ and $e n d_{1}$ need not be connected subsets of $Q$ - they must only be disjoint closed subsets of $Q$. We denote the set $e n d_{0} \cup e n d_{1}$ with the notation ends, and we also call the sets $e n d_{0}$ and $e n d_{1}$ the "ends" of $Q$.

\section{EXAMPLES}

We begin with the most obvious and arguably simplest example, the Smale horseshoe with crossing number 2. This system serves as a nice example that 


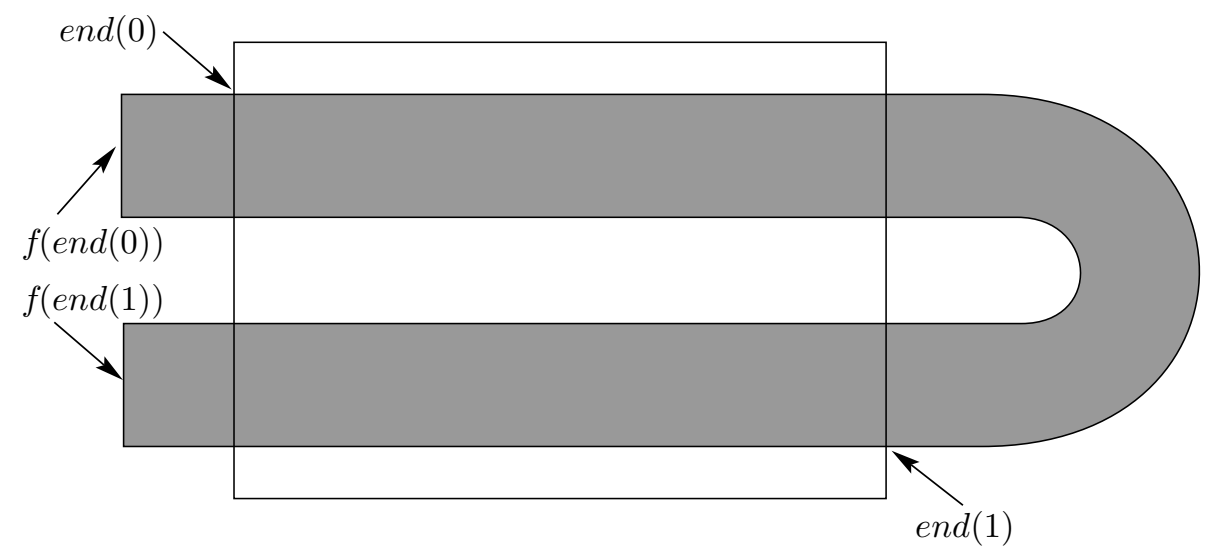

Figure 1. Smale horseshoe with crossing number $M=2$. The set $Q$ is the rectangle pictured, with end $d_{0}$ being the left boundary of $Q$ and $e n d_{1}$ being the right boundary of $Q$. The shaded region is the image $f(Q)$.

satisfies the horseshoe hypotheses $\boldsymbol{\Omega}$, and it is not hard to see how the ends and the set $Q$ should be chosen, nor what the natures of the connections and preconnections are. We then move to systems on the unit interval, to illustrate the necessity of the properties we require a map $f$ and associated set $Q$ have to satisfy the horseshoe hypotheses $\boldsymbol{\Omega}$ on $Q$. The last examples are less obvious applications of our results.

Example 3. Smale horseshoe with crossing number $M=2$. The set $Q$ and its image are illustrated in Figure 1 The horseshoe map is named $f$. Figure 2] shows $Q$ and two different connections, both of which are arcs, with their preconnections. Note that $Q$ itself is a connection, and that the vertical bands $\left(Q \cap f^{-1}(Q)\right)$ are preconnections in $Q$ (although they are not unique).

Example 4. Numerically obtained horseshoe. The topological horseshoe pictured in Figure 3 was obtained as a part of an investigation of a model of fluid flow past a sequence of cylinders. (See [SKGY, KSYG] for detailed discussions (mathematical and physical) of the model, and what can be proved rigorously when assumptions based on the numerical information are used.) The space for the Poincaré map of the flow given by the model is the plane. As is typical of such investigations, once the appropriate quadrangle $Q_{0}$ is found (that can be, and was, tedious), verifying horseshoe type behavior of the Poincaré map is usually straightforward. The map is an area preserving diffeomorphism on the plane (a property it inherits from the model), but verifying that the map is hyperbolic in $Q_{0}$ is quite difficult.

Example 5. Maps from the unit interval to itself. In these examples the entire space is the set $Q$. Let $X=[0,1]=Q$, end $d_{0}=\{0\}$, and $\operatorname{end}_{1}=\{1\}$. See Figure 4 for the graphs of these maps. Each map possesses exactly one connection, $[0,1]$. The first map, $f$, does not satisfy $\Omega$ because $[0,1]$ does not contain two disjoint preconnections, although it does contain two preconnections. The second map, $g$, satisfies $\Omega$ on $Q=[0,1]$ with crossing number $M=2$. Note that $g^{-1}(0)$ is the union of a subinterval $I_{1}$ containing 0 and subinterval $I_{3}$ containing 1 , while 


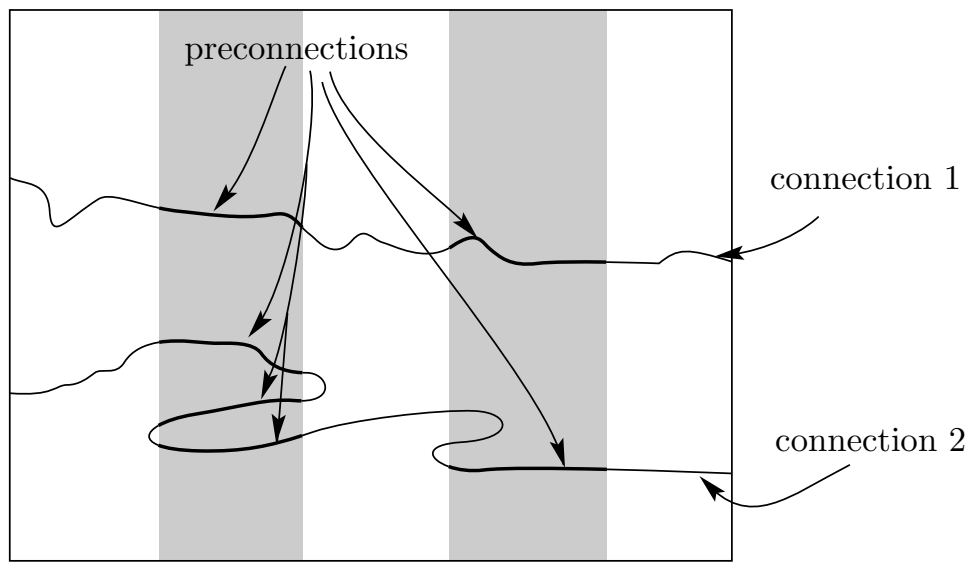

Figure 2. Connections and their preconnections for the Smale horseshoe. Any continuum that extends from end to $e n d_{1}$ in $Q$ is a connection. Two possible connections are pictured above. Both are arcs. Connection 1 has the minimum number of preconnections possible, namely 2, while connection 2 has 4 , due to the wiggly path it takes in travelling from end to $e n d_{1}$. The preconnections in this case are arcs in the grey-shaded regions which connect the two sides of a grey band.

$g^{-1}(1)$ is a subinterval $I_{2}$ in $(0,1)$. This map possesses two disjoint preconnections, the obvious choice for those being (1) the smallest subinterval of $[0,1]$ that contains a point of $I_{1}$ and a point of $I_{2}$, and (2) the smallest subinterval of [0,1] that contains a point of $I_{2}$ and a point of $I_{3}$. In this example, both $e n d_{0}$ and $e n d_{1}$ are contained in the interior of $g^{-1}\left(e n d_{0}\right)$. The third map, $h$, also satisfies $\boldsymbol{\Omega}$ with $M=2$. There are points $p<q$ in $(0,1)$ such that $h^{-1}(0)$ consists of two points, 0 and $q$, while $h^{-1}(1)$ consists of two points, $p$ and 1 . This map possesses two disjoint preconnections, the only choice for those being (1) the smallest subinterval of $[0,1]$ that contains 0 and $p$, and (2) the smallest subinterval of $[0,1]$ that contains $q$ and 1. The shift dynamics for Example 3(c) are implied by our results, but since there is no isolating neighborhood here (every open set containing the invariant Cantor set also contains periodic orbits not in the Cantor set), the shift dynamics do not follow from Szymczak's results $[\mathrm{S}$.

Example 6. Fewer conditions to satisfy. One reason locating the quadrangle $Q_{0}$ in Example 2 was so tedious was that it was necessary to find one that satisfied the following properties:

1. The quadrangle $Q_{0}$ must have sides labelled $s_{1}, s_{2}, s_{3}, s_{4}$ and vertices labelled $v_{1}, v_{2}, v_{3}, v_{4}$ such that $s_{i} \cap s_{i+1}=\left\{v_{i}\right\}$ for $i=1,2,3$ and $s_{4} \cap s_{1}=\left\{v_{4}\right\}$.

2. Two opposite sides, say $s_{1}$ and $s_{3}$, map outside $Q_{0}$ under $F$, i.e., $\left(F\left(s_{1}\right) \cap\right.$ $\left.F\left(s_{3}\right)\right) \cap Q_{0}=\varnothing$.

3. The image of the remaining two sides must not intersect those sides, i.e., $\left(F\left(s_{2}\right) \cap F\left(s_{4}\right)\right) \cap\left(s_{2} \cup s_{4}\right)=\varnothing$.

4. The set $Q \cap F(Q)$ must contain at least two connected components, each of which intersects both $s_{1}$ and $s_{3}$. 


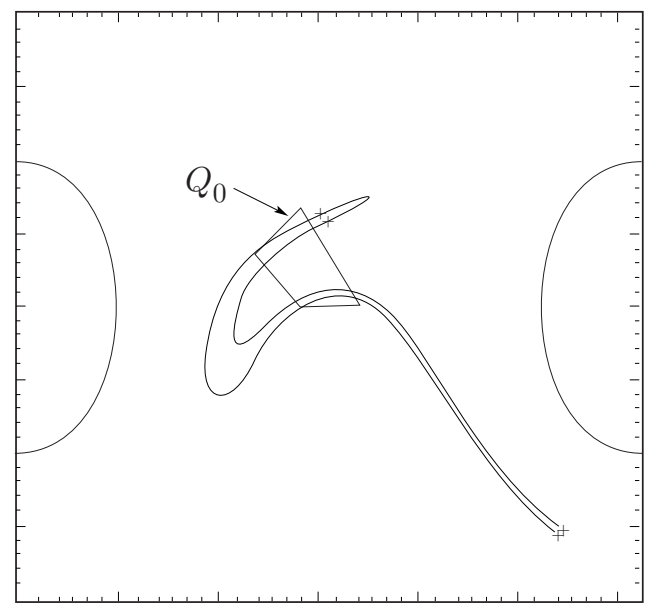

Figure 3. Horseshoe from a fluid-flow model. The figure shows a topological horseshoe obtained from the Poincaré map of a 2-dimensional area preserving fluid flow model. The crosses are the images of the vertices of the quadrilateral $Q_{0}$ under the action of the Poincaré map $F$. The map $F$ is an area preserving diffeomorphism on $\mathbf{R}^{2}$.

Figure 5illustrates a map $f$ on $\mathbf{R}^{2}$ and a rectangle $Q$ in $\mathbf{R}^{2}$ such that $f$ satisfies $\boldsymbol{\Omega}$ on $Q$ with crossing number $M=2$, but properties 2 and 3 above are not satisfied (and it is not necessary to choose $Q$ so very carefully). The map $f$ might well be a homeomorphism or even a diffeomorphism, but it most probably is not hyperbolic in $Q$. Even so, if $f$ is a continuous map, applying our theorem tells us that there is a "Cantor-like" invariant set $Q_{I}$ in $Q$ that factors over a two-sided two shift. However, without hyperbolicity, preimages of points in the two shift Cantor set need not be points - they need only be closed sets.

Example 7. It does not look like a horseshoe. The map $f$ indicated on the circular region $Q$ in Figure [6] satisfies $\boldsymbol{\Omega}$ on $Q$ with $M=2$. Note that the image of any continuum in $Q$ that intersects both $\operatorname{end}(0)$ and end(1), in other words, any connection, must have an image that intersects both $f(\operatorname{end}(0))$ and $f(\operatorname{end}(1))$, and this means that the connection itself must contain at least two disjoint preconnections.

Example 8. A more complicated example with $M=14$. We can think of $f: \mathbf{R}^{2} \rightarrow \mathbf{R}^{2}$ as a composition of maps on $Q$ ( $f$ is not specified outside $Q$-it does not matter as long as it is continuous).

1. First apply the map $f_{1}: Q \rightarrow S^{2}$ (where $S^{2}$ is the unit sphere in $\mathbf{R}^{3}$ ), with $f_{1}$ mapping the region between the boundary $\partial Q$ and end(1) (the outer annular region) to the point $(0,0,1), f_{1}$ mapping the region inside end(0) (the inner annular region) to the point $(0,0,-1)$, and $f_{1}$ being one-to-one on the rest of $Q$. We can do this so that the set $f_{1}(\operatorname{end}(0))$ is the set of all points $(x, y, z)$ in $S^{2}$ with $z \geq 0.999$, and $f_{1}(\operatorname{end}(1))$ is the set of all points $(x, y, z)$ in $S^{2}$ with $z \leq-0.999$. 


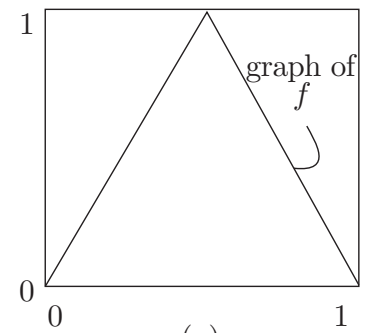

(a)

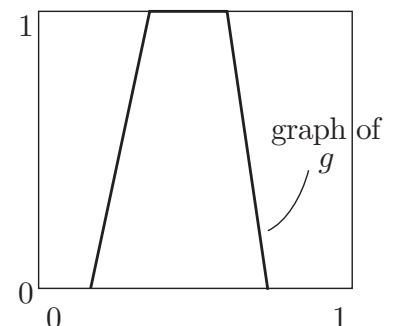

(b)

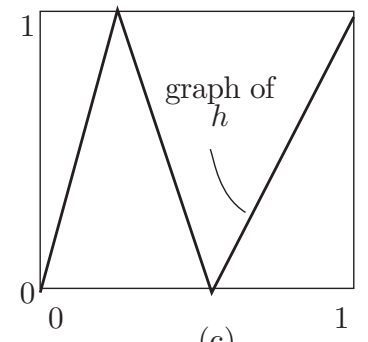

(c)

Figure 4. Interval maps. The graphs of three maps from $[0,1]$ to itself are pictured above. In these examples, $Q=[0,1]=X$, and $Q$ is the only connection.

2. Let $B=\left\{(x, y): x^{2}+y^{2} \leq 1\right\}$, and let $\mathbf{v}$ denote a vector in 3-space with direction chosen so that no line in the same direction as $\mathbf{v}$ intersects $f_{1}(\operatorname{end}(0)) \cup$ $f_{1}(\operatorname{end}(1))$ more than once. Then apply the map $f_{2}: S^{2} \rightarrow B$ by identifying points in $S^{2}$ that are on the same line $L$ in the same direction as $\mathbf{v}$. With an appropriate choice of $\mathbf{v}$, this can be done so that $f_{2}\left(f_{1}(\operatorname{end}(0)) \cup f_{1}(\operatorname{end}(1))\right) \subset$ $B^{\circ}=\left\{(x, y): x^{2}+y^{2}<1\right\}$. Note that no point $p$ in $B$ has more than two points in its preimage $f_{2}^{-1}(p)$.

3. Next apply a homeomorphism $f_{3}$ to contract $B$ in, say, the $y$-direction, and stretch it in the $x$-direction, and contract the sets $f_{2}\left(f_{1}(\operatorname{end}(0))\right.$, and $f_{2}\left(f_{1}(\operatorname{end}(1))\right)$ so that they have small diameter, obtaining a long thin tape.

4. Finally, apply the map $f_{4}$, which places the long thin band $f_{3} \circ f_{2} \circ f_{1}(Q)$ back in the plane, by looping it 8 times on $Q$ while crossing over end(0) and end(1), as shown.

Let $f=f_{4} \circ f_{3} \circ f_{2} \circ f_{1}$. Since any connection has an image that stretches from $f(\operatorname{end}(0))$ to $f(\operatorname{end}(1))$, it must contain at least 14 mutually disjoint preconnections. Note that one of the loops does not completely cross the inner annular region end(0), and that affects the crossing number $M=14$. See Figure 7 .

Example 9. Expanding balls. Suppose that $X=B_{n}=\left\{x \in \mathbf{R}^{n}: d(x, \mathbf{0}) \leq 1\right\}$ (where $\mathbf{0}$ denotes the origin and $n \geq 2$ ). Let balls $=\{I, I I, I I I, I V, V\}$ denote a mutually disjoint collection of smaller closed balls in $(X)^{\circ}=\left\{x \in \mathbf{R}^{n}: d(x, \mathbf{0})<1\right\}$, as pictured in Figure 8. The map $f$ takes each of the smaller balls homeomorphically onto $X$, and takes the boundary $\partial X=\left\{x \in \mathbf{R}^{n}: d(x, \mathbf{0})=1\right\}$ into itself. For simplicity, we also assume that if $\left\langle b_{i_{j}}\right\rangle_{j=0}^{\infty}$ is a sequence whose members are the small balls in $\{I, I I, I I I, I V, V\}$, then $\bigcap_{j=0}^{\infty} f^{-j}\left(b_{i_{j}}\right)$ is a single point. Now although there is an invariant Cantor set in $X$ on which the dynamics are conjugate to those of the two-sided 5 -shift, $f$ does not satisfy $\boldsymbol{\Omega}(X)$ for any choice of end(0) or $\operatorname{end}(1)$. With an appropriate choice of $Q$, end( 0$)$ and $\operatorname{end}(1)$, however, $f$ satisfies $\boldsymbol{\Omega}$. We carefully choose an $\operatorname{arc} Q$ which contains the invariant Cantor set: Start by choosing 6 disjoint $\operatorname{arcs} P_{J}, I \leq J \leq V I$, in $\overline{X \backslash(\bigcup b a l l s)}$ as follows. Choose $P_{I}$ to be an arc which has a one point intersection with $\partial X$ and a one point intersection with $I$; for $I<J<V I$, choose $P_{J}$ to be an arc which has a one point intersection with $J-I$ and a one point intersection with $J$; and choose $P_{V I}$ to be an arc which has a one point intersection with $V$ and a one point intersection with $\partial X$. Let $P=\bigcup_{j=I}^{I V} P_{i}$ 


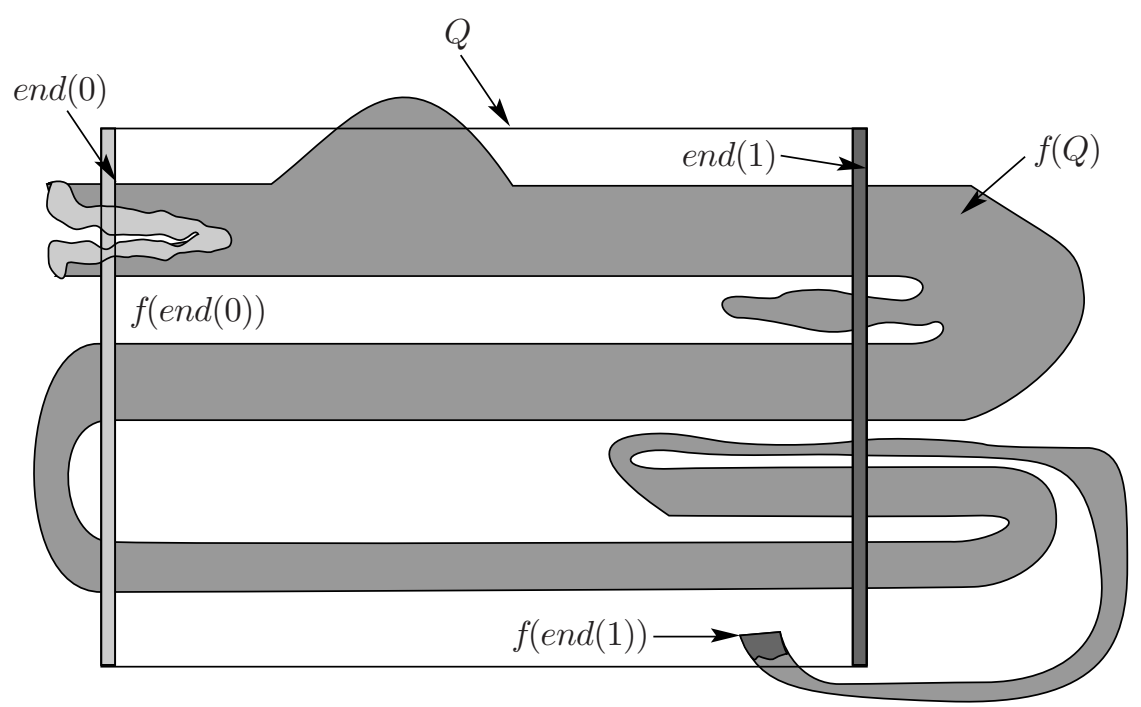

Figure 5. No side or end conditions. If $Q$ is the rectangular region, the map $f$ illustrated above satisfies $\boldsymbol{\Omega}(Q)$ with crossing number $M=2$. The sets $\operatorname{end}(0)$ and $\operatorname{end}(1)$ are strips along the left and right boundaries, respectively. Note that the connected component of $Q \cap f(Q)$ does not "count' since no subinterval of the top boundary of $Q \cap f^{-1}(Q)$ that is contained in maps to the top boundary of the top connected component.

and let $P^{\prime}=\bigcup_{j=I}^{V} P_{i}$. Next define $Q_{0}=\bigcup_{j=I}^{V I} P_{i}$. Then let $Q_{1, b}=f^{-1}\left(Q_{0}\right) \cap b$ for $b \in$ balls. This can be done so that $Q_{1}=\bigcup_{b \in \text { balls }} Q_{1, b}$ does not $Q_{1, b}=f^{-1}\left(Q_{0}\right) \cap b$ for $b \in$ balls. This can be done so that $Q_{1}=\bigcup_{b \in \text { balls }} Q_{1, b}$ does not intersect $Q_{0}$. To make sure that the end result is connected, for $b \in$ balls choose 2 disjoint $\operatorname{arcs} A_{b, 1}$ and $A_{b, 2}$ in $\partial b$ such that $A_{b, 1} \cap P_{b}$ consists of one point, $A_{b, 2} \cap P_{b+I}$ consists of one point, $A_{b, 1} \cap\left(f^{-1}\left(P_{I}\right) \cap b\right)$ consists of one point, and $A_{b, 2} \cap\left(f^{-1}\left(P_{V I}\right) \cap b\right)$ consists of one point. If $A=\bigcup\left\{A_{b, i}: b \in\right.$ balls, $\left.i=1,2\right\}, L_{0}=A \cup Q_{0} \cup Q_{1}$ consists of 6 disjoint arcs. For $n>1$, let $L_{n}=f^{-n}\left(L_{0}\right)$ and let $\widetilde{Q}=\overline{\bigcup_{n \geq 0} L_{n}}$. By construction, $\widetilde{Q}$ is an arc. Finally we shorten $\widetilde{Q}$ to make the set $Q$ be an irreducible between some point in the "interior" of $Q$ : define $Q$ to be $\widetilde{Q} \backslash P_{V I}$. Suppose $e n d_{0}=\partial X \cap Q$, and $e n d_{1}=\partial V \cap f^{-1}\left(P_{V I}\right)$. Thus, end $d_{0}$ is a single point on the boundary $\partial X$, end is a single point on the boundary $\partial V$, and $f$ satisfies the horseshoe hypotheses $\boldsymbol{\Omega}$ on $Q$ relative to ends $e n d_{0}$ and $e n d_{1}$ with crossing number 5 .

Example 10. The invariant set may contain no periodic points. Suppose that $f: \mathbf{R}^{2} \rightarrow \mathbf{R}^{2}$ is the canonical Smale horseshoe map with crossing number 2 (as in the first example), and $r: S^{1} \rightarrow S^{1}$ is a rotation by an irrational number. If $Q$ denotes the rectangular region in Example 1, with end end(0) and end(1) chosen to be the vertical sides of $Q$, then the map $f \times r: \mathbf{R}^{2} \times S^{1} \rightarrow \mathbf{R}^{2} \times S^{1}$ satisfies $\boldsymbol{\Omega}\left(Q \times S^{1}\right)$ relative to ends end $(0) \times S^{1}$ and $\operatorname{end}(1) \times S^{1}$ with crossing number 2 . However, there are no periodic points in $Q \times S^{1}$ under the action of $f \times r$. 


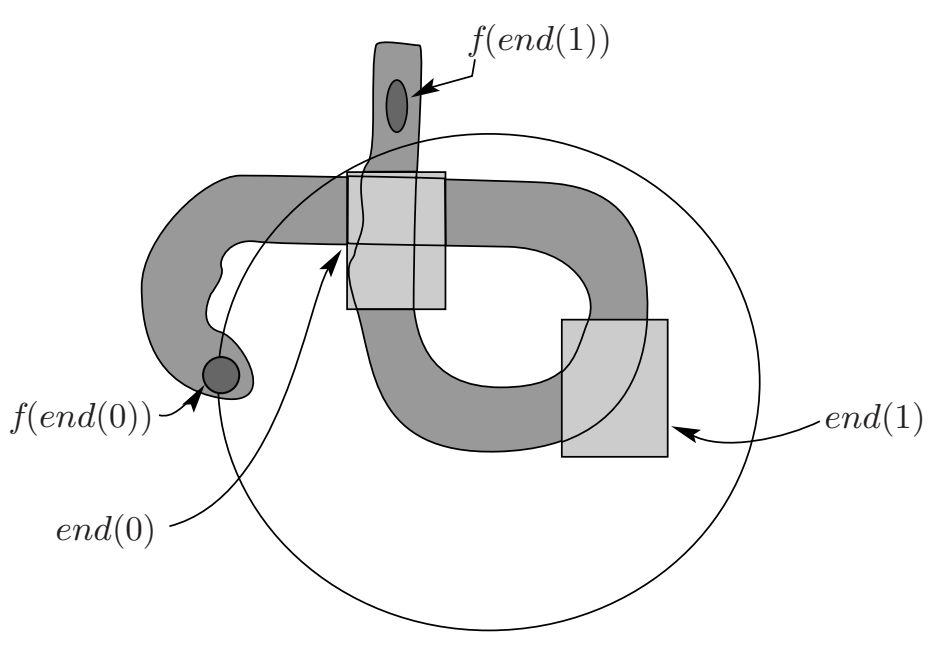

FiguRE 6. Stretched, squeezed and folded disk. In this example, the ends are in the interior of $Q$. The map $f$ is not a homeomorphism, because the image "crosses itself," i.e., there are points $x$ in $f(Q) \cap Q$ such that $f^{-1}(x)$ contains at least two points.

\section{The Results}

Recall that in the results that follow, we consider the topology on $Q$ only. The sets in which we are interested are all contained in $Q$; this restriction to $Q$ avoids our having to add repeatedly " $\cap Q$ " to the sets discussed.

Since $Q$ is locally connected we may choose the distance function $d$ on $Q$ so that for $x \in Q, D_{\varepsilon}(x)=\{y \in Q: d(x, y)<\varepsilon\}$ is connected as long as $D_{\varepsilon}(x)$ is contained in the component of $Q$ that contains $x$. (See $[\mathrm{Ku}]$ for a proof.)

Lemma 11. Suppose that $f: X \rightarrow X$ is continuous, $f$ satisfies the horseshoe hypotheses $\Omega$ on the set $Q$ with crossing number $M$ relative to ends end $d_{0}$ and end . $_{1}$. Then there is $\varepsilon>0$ with the following properties:

1. If Com is a component of $Q$, either $E_{0}=f^{-1}\left(\right.$ end $\left._{0}\right) \backslash D_{\varepsilon}$ (ends) separates Com between end $d_{0}$ and end $d_{1}$; or $E_{1}=f^{-1}\left(\right.$ end $\left._{1}\right) \backslash D_{\varepsilon}($ ends $)$ separates Com between end $d_{0}$ and $e n d_{1}$.

2. The set $E=f^{-1}($ ends $) \backslash D_{\varepsilon}$ (ends) separates $Q$ between end $d_{0}$ and end $d_{1}$.

3. If $D=\left\{D_{0}, D_{1}, \ldots, D_{n}\right\}$ is a tiling chain such that

(a) each link $D_{i}$ of $D$ is the closure of a nonempty open set;

(b) $\bigcup D \subset Q$ is a continuum;

(c) $D_{0} \cap e n d_{0} \neq \varnothing$;

(d) $D_{n} \cap e n d_{1} \neq \varnothing$;

(e) for $0<i<n, D_{i} \cap D_{\varepsilon}($ ends $)=\varnothing$;

(f) for $0 \leq i \leq n,\left(D_{i}^{\circ} \backslash D_{\varepsilon}(\right.$ ends $\left.)\right) \cap f^{-1}($ ends $)=\varnothing$; and

(g) either

i. for $0 \leq i<n$, the right boundary of the link $D_{i}$ is contained in $f^{-1}\left(\right.$ end $\left._{0}\right)$, or the right boundary of the link $D_{i}$ is contained in $f^{-1}\left(e n d_{1}\right)$, and 
ii. for $0<i \leq n$, the left boundary of the link $D_{i}$ is contained in $f^{-1}\left(\right.$ end $\left._{0}\right)$, or the left boundary of the link $D_{i}$ is contained in $f^{-1}\left(e n d_{1}\right)$,

then $D$ has at least $2 M-1$ links, and there is a mutually disjoint subcollection $D^{\prime}$ of $D$ of which each member contains a preconnection and which contains at least $M$ members.

Proof. Note that no component of $f^{-1}\left(e n d_{0}\right)$ contains both a point of end $d_{0}$ and a point of end . There is some $\varepsilon>0$ such that $\overline{D_{2 \varepsilon}\left(e n d_{0}\right)} \cap \overline{D_{2 \varepsilon}\left(e n d_{1}\right)}=\varnothing$, $\overline{D_{2 \varepsilon}\left(f^{-1}\left(e n d_{0}\right)\right)} \cap \overline{D_{2 \varepsilon}\left(f^{-1}\left(e n d_{1}\right)\right)}=\varnothing$, and such that if $x \in Q$, then $\overline{D_{\varepsilon}(x)}$ contains no preconnection. Suppose that Com is a component of $Q$.

To prove the first property, that either $E_{0}=f^{-1}\left(e n d_{0}\right) \backslash D_{\varepsilon}(e n d s)$ separates Com between $e n d_{0}$ and $e n d_{1}$, or $E_{1}=f^{-1}\left(e n d_{1}\right) \backslash D_{\varepsilon}(e n d s)$ separates Com between $e n d_{0}$ and $e n d_{1}$, suppose that $E_{0}=f^{-1}\left(e n d_{0}\right) \backslash D_{\varepsilon}(e n d s)$ does not separate Com between end $d_{0}$ and $e n d_{1}$, and consider $E_{1}=f^{-1}\left(e n d_{1}\right) \backslash D_{\varepsilon}(e n d s)$. If $E_{1}=$ $f^{-1}\left(e n d_{1}\right) \backslash D_{\varepsilon}(e n d s)$ does not separate Com between end $d_{0}$ and end either, then there is an $\operatorname{arc} A$ irreducible from end $d_{0}$ to end $d_{1}$ in $C o m$ such that $A \cap \overline{D_{\varepsilon}\left(e n d_{0}\right)} \subset \overline{D_{\varepsilon}\left(x_{0}\right)}$ for some $x_{0} \in e n d_{0}, A \cap \overline{D_{\varepsilon}\left(e n d_{1}\right)} \subset \overline{D_{\varepsilon}\left(x_{1}\right)}$ for some $x_{1} \in e n d_{1}, A \backslash\left(D_{\varepsilon}\left(e n d_{0}\right) \cup D_{\varepsilon}\left(e n d_{1}\right)\right)=A^{\prime}$ is an arc, and $A^{\prime} \subset C o m \backslash E_{1}$. But then $A \cap \overline{D_{+} \varepsilon\left(e n d_{0}\right)}$ contains no preconnection, $A \cap \overline{D_{\varepsilon}\left(e n d_{1}\right)}$ contains no preconnection, and $A \backslash\left(D_{\varepsilon}\left(e n d_{0}\right) \cup D_{\varepsilon}\left(e n d_{1}\right)\right)$ contains no preconnection.

Since $A$ is a connection, it must contain at least $M$ mutually disjoint preconnections. The only way this can happen is for $A \cap \overline{D_{\varepsilon}\left(e n d_{0}\right)}$ to intersect $f^{-1}\left(e n d_{1}\right), A \cap \overline{D_{\varepsilon}\left(e n d_{1}\right)}$ to intersect $f^{-1}\left(e n d_{1}\right)$, and $A^{\prime}$ to intersect $f^{-1}\left(e n d_{0}\right)$. Further, this means that $A \cap \overline{D_{\varepsilon}\left(e n d_{0}\right)}$ does not intersect $f^{-1}\left(\right.$ end $\left._{0}\right)$ (because $\left.\overline{D_{2 \varepsilon}\left(f^{-1}\left(e n d_{0}\right)\right)} \cap \overline{D_{2 \varepsilon}\left(f^{-1}\left(e n d_{1}\right)\right)}=\varnothing\right), A \cap \overline{D_{\varepsilon}\left(e n d_{1}\right)}$ does not intersect $f^{-1}\left(\right.$ end $\left._{0}\right)$, and $M=2$. Since $E_{0}$ does not separate $C o m$ between $e n d_{0}$ and $e n d_{1}$, there is an $\operatorname{arc} \widehat{A}$ with endpoints the endpoints of $A^{\prime}$, which is contained in $C o m \backslash E_{0}$. Then $\left(A \cap\left(D_{\varepsilon}\left(e n d_{0}\right) \cup D_{\varepsilon}\left(e n d_{1}\right)\right)\right) \cap \widehat{A}$ is an arc and a connection, but it fails to intersect $f^{-1}\left(e n d_{0}\right)$, so that it contains no preconnection. This is a contradiction. Thus, either $E_{0}$ separates $C o m$ between $e n d_{0}$ and $e n d_{1}$, or $E_{1}$ does, and the first property is proved. The second property follows immediately from the first.

To prove the third property, suppose that $D=\left\{D_{0}, D_{1}, \ldots, D_{n}\right\}$ is a tiling chain satisfying the conditions listed in the statement. Since $\bigcup D$ is a connection, we can choose a continuum $K=\bigcup_{i=0}^{n} K_{i}$ irreducible from $e n d_{0}$ to $e n d_{1}$ with the following properties:

1. The set $K_{0}$ is a continuum in $D_{0}$ irreducible from end to $D_{1}$.

2. The set $K_{n}$ is a continuum in $D_{n}$ irreducible from $D_{n-1}$ to $e n d_{1}$.

3. For $0<i<n, K_{i}$ is a continuum in $D_{i}$ irreducible from $D_{i-1}$ to $D_{i+1}$.

4. For $0 \leq i<n, K_{i} \cap K_{k+1} \neq \varnothing$.

Then $K$ must contain $M$ mutually disjoint irreducible preconnections $\left\{P_{\alpha_{0}}, P_{\alpha_{1}}\right.$, $\left.P_{\alpha_{2}}, \ldots, P_{\alpha_{M}}\right\}$. Because of the properties of the chain $D$ and of the continuum $K$, we can assume that the indexing set $\left\{\alpha_{0}, \alpha_{1}, \alpha_{2}, \ldots, \alpha_{M}\right\}$ is a subsequence of $\{0,1, \ldots, n\}$, and that $P_{\alpha_{1}} \subset D_{\alpha_{i}}$. Further, it must be the case that for $0<$ $\alpha_{i}<n, P_{\alpha_{i}}=K_{\alpha_{i}}$; that if $\alpha_{1}=0$, then $P_{\alpha_{M}} \subset K_{\alpha_{M}}, P_{\alpha_{M}} \cap D_{\varepsilon}\left(e \operatorname{end}_{1}\right) \neq \varnothing$, and $P_{\alpha_{M}} \cap D_{n-1} \neq \varnothing$. Since $P_{\alpha_{i}} \cap P_{\alpha_{i}+1}=\varnothing, \alpha_{i+1}-\alpha_{i} \geq 2$. Thus, $D$ must have a minimum of $2 M-1$ links, and it must contain a subcollection $D^{\prime}$ of $M$ mutually disjoint links each of which contain a preconnection. 


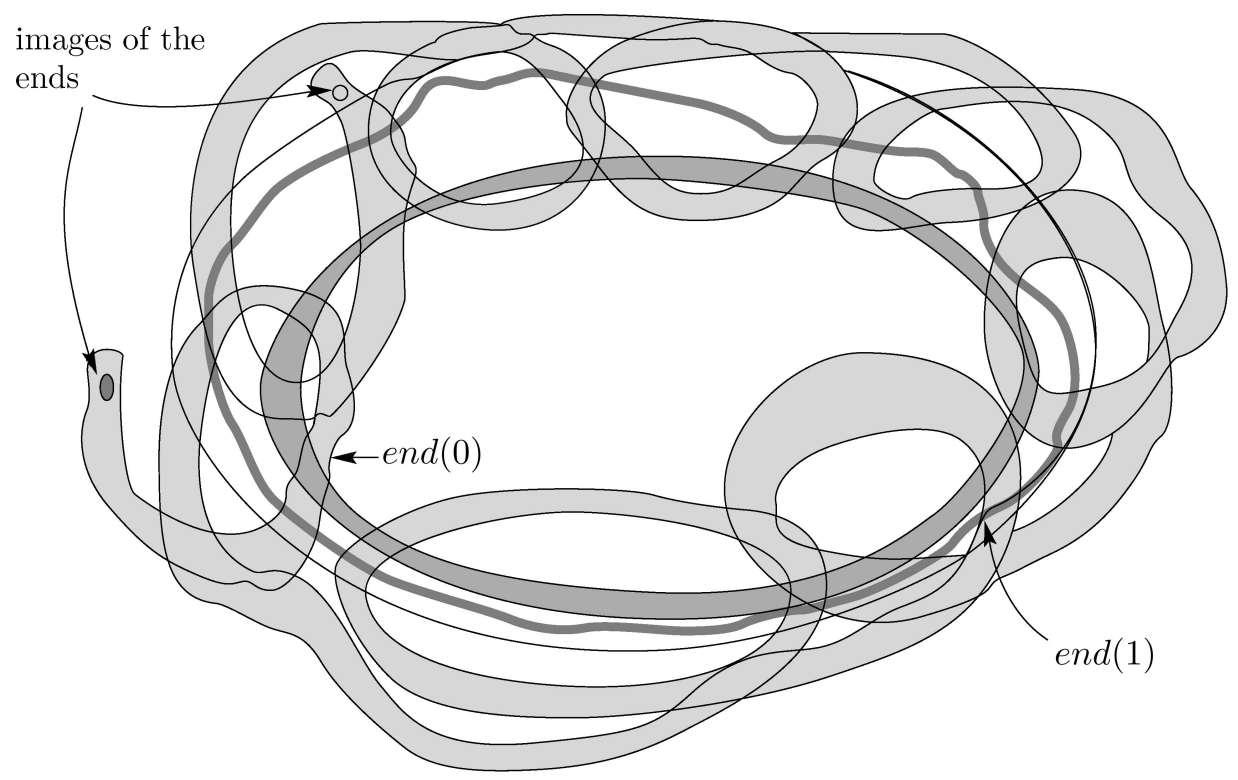

FiguRE 7. Stretch, squeeze and loop 8 times. The elliptical region is the set $Q$, with $\operatorname{end}(0)$ and $\operatorname{end}(1)$ the two annular regions in the interior of $Q$, as indicated. The dark shaded winding region is the image of $Q$ under $f$, with the images of the ends the small disk-like regions indicated.

Lemma 12. Suppose that $f: X \rightarrow X$ is continuous, and $f$ satisfies the horseshoe hypotheses $\Omega$ on $Q$ relative to ends end $d_{0}$ and end $d_{1}$ and has crossing number $M$. Then there is a collection $S=\left\{S_{1}, S_{2}, \ldots, S_{M}\right\}$ of $M$ mutually disjoint closed neighborhoods of $Q$ having the property that $f\left(S_{i}\right) \cap S_{j} \neq \varnothing$ for each pair $(i, j) \in$ $\{1,2, \ldots, M\}^{2}$. Thus $\mathcal{S}$ is a set of symbol sets for $Q$.

Proof. Suppose Com is a component of $Q$. Without loss of generality, suppose that $Q_{1} \subset f^{-1}\left(e n d_{1}\right)$ separates $C o m \backslash E_{1}$ between $e n d_{0}$ and $e n d_{1}$ (using the $\varepsilon, E_{1}, E_{0}$ of the last lemma). The boundary of each component of $C o m \backslash E_{1}$ is contained in $f^{-1}\left(e n d_{1}\right)$, but it may not be the case that

$$
\overline{\bigcup\left\{C: C \text { is a component of } C o m \backslash E_{1}\right\}}=C o m,
$$

because it is possible that $E_{1}^{\circ} \neq \varnothing$. Then consider the collection $\mathcal{S}_{\text {Com }}$ consisting of all members of $\mathcal{S}_{1 \text { Com }} \cup \mathcal{S}_{2 \text { Com }} \cup \mathcal{S}_{3 \text { Com }}$, where $\mathcal{S}_{1 \text { Com }}=\{\bar{C}: C$ is a component of $C o m \backslash E_{1}$, and $f^{-1}\left(e n d_{0}\right)$ separates $\left.C\right\}, \mathcal{S}_{3 C o m}=\left\{\bar{C}: c\right.$ is a component of $C o m \backslash E_{1}$, and $f^{-1}\left(e n d_{0}\right) \cap C \neq \varnothing$, and $f^{-1}\left(e n d_{0}\right)$ does not separate $\left.C\right\}$, and $\mathcal{S}_{3 \mathrm{Com}}=\{\bar{C}: C$ is a component of $C o m \backslash \overline{\left(\bigcup\left(\mathcal{S}_{1 C o m} \cup \mathcal{S}_{2 C o m}\right)\right)}$. Because Com is locally connected, and $\partial \bar{C}$, for $\bar{C} \in \mathcal{S}_{1 \text { Com }}$, is continued in $f^{-1}\left(e n d_{1}\right)$, each member of $\mathcal{S}_{1 \mathrm{Com}} \cup \mathcal{S}_{2 \mathrm{Com}}$ must contain an open set of diameter $\varepsilon$. Thus, $\mathcal{S}_{1 \text { Com }} \cup \mathcal{S}_{2 \text { Com }}$ is finite. 


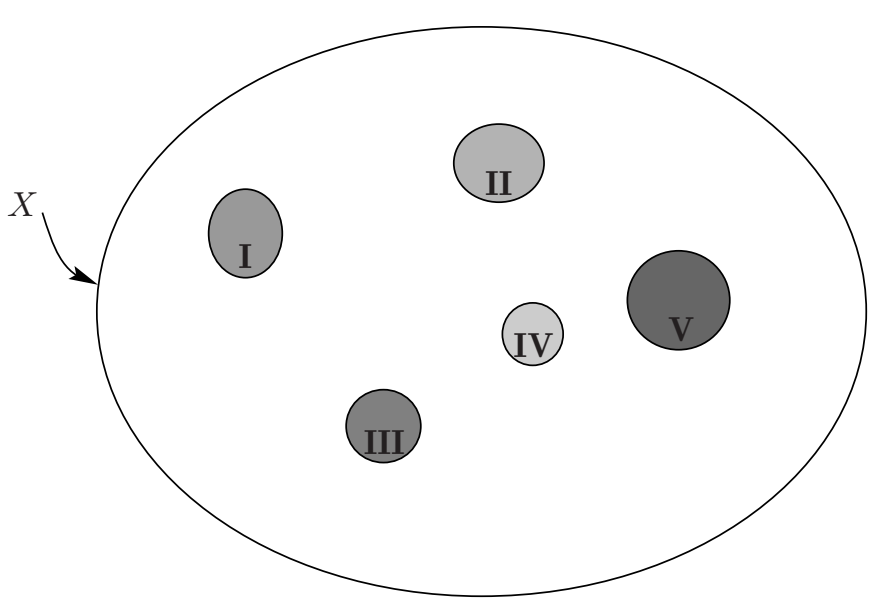

Figure 8. Expanding balls. Suppose that $X$ is homeomorphic to a closed $n$-ball, and $I, I I, I I I, I V$, and $V$ are mutually disjoint closed $n$-balls contained in the interior of $X$. The map $f: X \rightarrow X$ maps each interior ball homeomorphically onto $X$, and points in the complement of the $n$-balls to the boundary $\partial X$.

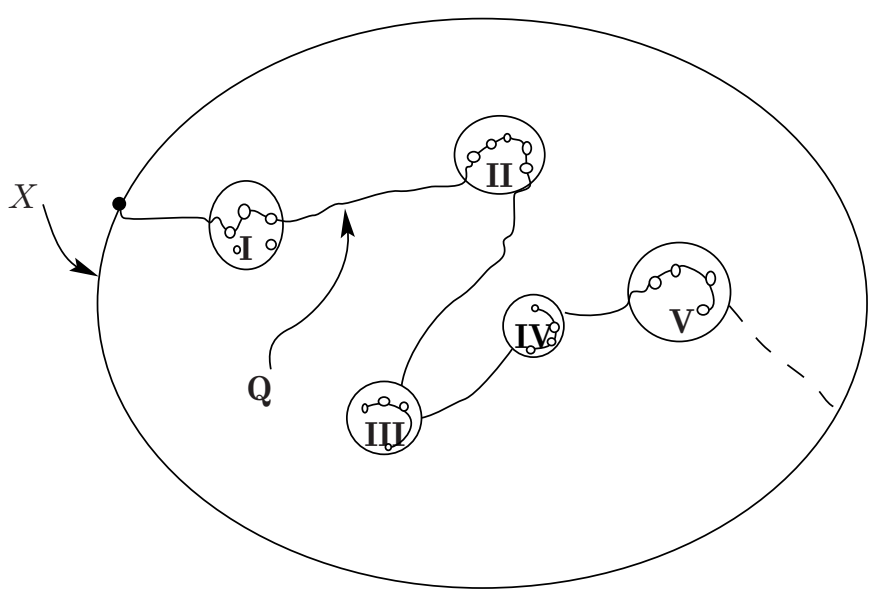

Figure 9. Choosing $Q$. The figure shows how $Q$ can be chosen in order to satisfy the horseshoe hypotheses and pick up the 5-shift dynamics. Here, as long as each component of the invariant set is a single point $Q$ can be a carefully chosen arc.

Construct inductively the tiling chain $\mathcal{T}_{\text {Com }}^{\prime}=\left\{T_{C o m, 0}^{\prime}, T_{C o m, 1}^{\prime}, \ldots, T_{C o m, N}^{\prime}\right\}$ as follows: Let $T_{C o m, 0}^{\prime}=\bigcup\left\{\bar{C}: C \in \mathcal{S}_{C o m}\right.$ and $C \cap$ end $\left.d_{0} \neq \varnothing\right\}$. Let $T_{C o m, 1}^{\prime}=$ $\bigcup\left\{\bar{C}: C \in \mathcal{S}_{C o m}, \bar{C} \cap T_{C o m, 0}^{\prime} \neq \varnothing\right.$ and $\left.C \cap\left(T_{C o m, 0}^{\prime}\right)^{\circ}=\varnothing\right\}$. Let

$$
T_{C o m, 2}^{\prime}=\bigcup\left\{\bar{C}: C \in \mathcal{S}_{C o m}, \bar{C} \cap\left(T_{C o m, 0}^{\prime} \cup T_{C o m, 1}^{\prime}\right) \neq \varnothing,\right.
$$

and

$$
\left.C \cap\left(T_{C o m, 0}^{\prime} \cup T_{C o m, 1}^{\prime}\right)^{\circ}=\varnothing\right\}
$$


Let $T_{C o m, 3}^{\prime}=\bigcup\left\{C: C \in \mathcal{S}_{C o m}, \bar{C} \cap\left(T_{C o m, 0}^{\prime} \cup T_{C o m, 1}^{\prime} \cup T_{C o m, 2}^{\prime}\right) \neq \varnothing\right.$, and $C \cap$ $\left.\left(T_{\text {Com }, 0}^{\prime} \cup T_{\text {Com }, 1}^{\prime} \cup T_{\text {Com }, 2}^{\prime}\right)^{\circ}=\varnothing\right\}$. Since $\mathcal{S}_{1 \text { Com }} \cup \mathcal{S}_{2 \text { Com }}$ is finite, and members of $\mathcal{S}_{3 \mathrm{Com}}$ must intersect some member of $\mathcal{S}_{1 \mathrm{Com}} \cup \mathcal{S}_{2 \mathrm{Com}}$, but do not intersect any other member of $\mathcal{S}_{3 \mathrm{Com}}$ except in points contained in $\mathcal{S}_{1 \text { Com }} \cup \mathcal{S}_{2 \text { Com }}$, the construction must be a finite process. Continue this finite process until each member of $\mathcal{S}_{\text {Com }}$ is in some member of $\mathcal{T}_{\text {Com }}^{\prime}$, thereby obtaining, for some positive integer $N$, the desired chain $\mathcal{T}_{\text {Com }}^{\prime}=\left\{T_{C o m, 0}^{\prime}, T_{C o m, 1}^{\prime}, \ldots, T_{C o m, N}^{\prime}\right\}$. Note that $\bigcup \mathcal{T}_{C o m}^{\prime}=C o m$, and that $\partial T_{C o m, l}^{\prime} \subset f^{-1}\left(e n d_{1}\right)$ for each $l$.

We can define a partial ordering $\prec$ on the members of $\mathcal{S}_{C o m}$ by defining $C \prec C^{\prime}$ in $\mathcal{S}_{C o m}$ if there exists a tiling chain $D=\left\{D_{0}, D_{1}, \ldots, D_{N^{\prime}}\right\} \subset \mathcal{S}_{C o m}$ such that for some $i<j, C=D_{i} \subset T_{C o m, i}^{\prime}$ and $C^{\prime}=D_{j} \subset T_{C o m, j}^{\prime}$. Note that if $C \in \mathcal{S}_{C o m}$, there is some member $C^{b}$ of $\mathcal{S}_{C o m}$ such that $C^{b} \prec C$ and $C^{b} \cap e n d_{0} \neq \varnothing$. For $1 \leq i \leq N$, and $C \in \mathcal{S}_{C o m}$, we define the left boundary $\partial_{l} C$ of $C$ to be $C \cap T_{C o m, i-1}^{\prime}$. For $0 \leq i \leq N-1$, and $C \in \mathcal{S}_{C o m}$, we define the right boundary $\partial_{r} C$ of $C$ to be $C \cap T_{C o m, i+1}^{\prime}$.

As for $E_{1}$ and $f^{-1}\left(e n d_{1}\right)$, it is possible that $f^{-1}\left(e n d_{0}\right)$ has nonempty interior in $Q$. Next we "split" each member $D$ of $\mathcal{S}_{1 \text { Com }}$ into continua and replace $D$ with those sets as follows: For each $D \in \mathcal{S}_{1 \text { Com }}$ such that $D \cap\left(e n d_{0} \cup e n d_{1}\right)=\varnothing$, let $\mathcal{D}_{l b}=\left\{\bar{C}: C\right.$ is a component of $D \backslash f^{-1}\left(e n d_{0}\right)$ and $\left.D \cap \partial D_{l} \neq \varnothing\right\}$. Because $Q$ is locally connected, the components $D$ of $\mathcal{D}_{l b}$ that intersect both $f^{-1}\left(\right.$ end $\left._{0}\right)$ and $f^{-1}\left(e n d_{1}\right)$ form a finite collection. There may be infinitely many components of $\mathcal{D}_{l b}$ that intersect only $f^{-1}\left(e n d_{1}\right)$, but these must be 'small' and 'do not matter' (relative to this construction). Let $\mathcal{D}_{r b}=\left\{\bar{C}: C\right.$ is a component of $\left.D \backslash\left(\overline{\bigcup D_{l b}}\right)\right\}$. Again, $\mathcal{D}_{r b}$ may be infinite, but has only finitely many components that 'matter', namely those that intersect both $f^{-1}\left(e n d_{0}\right)$ and $f^{-1}\left(e n d_{1}\right)$. For each $D \in \mathcal{S}_{1 \text { Com }}$ such that $D \cap e n d_{0} \neq \varnothing$, let $\mathcal{D}_{r b}=\left\{\bar{C}: C\right.$ is a component of $D \backslash f^{-1}\left(e n d_{0}\right)$ and $\left.D \cap \partial D_{r} \neq \varnothing\right\}$ and let $\mathcal{D}_{l b}=\left\{\bar{C}: C\right.$ is a component of $\left.D \backslash\left(\overline{\bigcup D_{r b}}\right)\right\}$. For each $D \in \mathcal{S}_{1 \text { Com }}$ such that $D \cap e n d_{1} \neq \varnothing$, let $\mathcal{D}_{l b}=\left\{\bar{C}: C\right.$ is a component of $D \backslash f^{-1}\left(e n d_{0}\right)$ and $\left.D \cap \partial D_{l} \neq \varnothing\right\}$ and let $\mathcal{D}_{r b}=\left\{\bar{C}: C\right.$ is a component of $\left.D \backslash\left(\overline{\bigcup D_{l b}}\right)\right\}$.

Note that for each $D \in \mathcal{S}_{1 \text { Com }}, D=\left(\overline{\bigcup \mathcal{D}_{l b}}\right) \cup\left(\overline{\bigcup \mathcal{D}_{r b}}\right)$. Let $\mathcal{C}_{C o m}=\mathcal{S}_{2 \text { Com }} \cup$ $\mathcal{S}_{3 C o m} \cup\left\{\mathcal{D}_{l b}: D \in \mathcal{S}_{C o m}\right\} \cup\left\{\mathcal{D}_{l b}: D \in \mathcal{S}_{\text {Com }}\right\}$.

We can "extend" the partial ordering $\prec$ on $\mathcal{S}_{C o m}$ to a partial ordering $\triangleleft$ on the members of $\mathcal{C}_{\mathrm{Com}}$ in a natural way, and we can also define the left boundary for members of $\mathcal{C}_{\mathrm{Com}}$ that do not contain a point of $e n d_{0}$, as well as the right boundary for members of $\mathcal{C}_{\text {Com }}$ which are followed by other members of $\mathcal{C}_{C o m}$ (relative to the partial ordering $\triangleleft)$. Let $\mathcal{D}_{\text {Com }}=\left\{\underline{D}=\left\{D_{0}, D_{1}, \ldots, D_{n^{\prime}}\right\}: \underline{D}\right.$ is a tiling chain, each link $D_{i}$ in $\underline{D}$ is a member of $\left.\mathcal{C}_{C o m}\right\}$, and $\mathcal{D}_{\text {Com }}^{\prime}=\left\{\underline{D}=\left\{D_{0}, D_{1}, \ldots, D_{N^{\prime}}\right\}: \underline{D} \in\right.$ $\mathcal{D}_{C o m}^{\prime}, D_{0}$ contains a point of $e n d_{0}, D_{N^{\prime}}$ contains a point of $e n d_{1}$, and no link $D_{i}$, $i<N^{\prime}$, contains a point of $\left.e n d_{1}\right\}$.

Then $\mathcal{C}_{\text {Com }}$ has the following properties:

1. Each nonempty left boundary of a member of $\mathcal{C}_{\text {Com }}$ that does not intersect $e n d_{0}$ is contained in either $f^{-1}\left(e n d_{0}\right)$ or it is contained in $f^{-1}\left(e n d_{1}\right)$.

2. Each nonempty right boundary of a member of $\mathcal{C}_{\mathrm{Com}}$ is contained in either $f^{-1}\left(e n d_{0}\right)$ or it is contained in $f^{-1}\left(e n d_{1}\right)$.

3. If $D \in \mathcal{D}_{\text {Com }}$, then no two consecutive links of $D$ have both the (nonempty) left and (nonempty) right boundaries contained in $f^{-1}\left(e n d_{0}\right)$. 
If $\underline{D}=\left\{D_{0}, D_{1}, \ldots, D_{N^{\prime}}\right\}$ is a tiling chain composed of members of $\mathcal{C}_{\text {Com }}$ and $D_{0}$ intersects $e n d_{0}, D_{N^{\prime}}$ intersects $e n d_{1}$, but no link $D_{i}, 0<i<N^{\prime}$, intersects ends, then we call $\underline{D}$ an irreducible end $d_{0}-e n d_{1} \mathcal{C}_{C o m}$ tiling chain. If $C \in \mathcal{C}_{C o m}$ such that

1. $C \cap\left(e n d_{0} \cup e n d_{1}\right)=\varnothing$, and each continuum in $C$ which intersects the nonempty left boundary of $C$ and the nonempty right boundary of $C$ contains a preconnection,

2. $C \cap e n d_{1} \neq \varnothing$, and each continuum in $C$ which intersects end 0 and the nonempty right boundary of $C$ contains a preconnection, or

3. $C \cap e n d_{1} \neq \varnothing$, and each continuum in $C$ which intersects the nonempty left boundary of $C$ and $e n d_{1}$ contains a preconnection,

then we call $C$ a preconnection link in $\mathcal{C}_{\text {Com }}$. Note that if $C$ is a preconnection link, then each continuum in $C$ irreducible from $f^{-1}\left(e n d_{0}\right)$ to $f^{-1}\left(e n d_{1}\right)$ is a subset of $Q \cap$ $f^{-1}(Q)$. To each irreducible $e n d_{0^{-}} e n d_{1} \mathcal{C}_{C o m}$ tiling chain $\underline{D}=\left\{D_{0}, D_{1}, \ldots, D_{N^{\prime}}\right\}$, we associate a connection $K_{\underline{D}}$ constructed as follows:

1. Let $L_{0}=e n d_{0}$ and for $0<i \leq N^{\prime}$, let $L_{i}=\partial D_{i-1} \cap \partial D_{i}$. For $0 \leq i<N^{\prime}$, let $R_{i}=\partial D_{i+1} \cap \partial D_{i}$, and let $R_{N^{\prime}}=e n d_{1}$.

2. The continuum $K_{\underline{D}}$ is a union of continua contained in the links $D_{i}$ of $\underline{D}$, that is, $K_{\underline{D}}=\bigcup_{i=0}^{N^{\prime}} K_{\underline{D}, i}$, where each $K_{\underline{D}, i}$ is a continuum contained in $D_{i}$ which intersects both $L_{i}$ and $R_{i}$.

3. If for some $i, D_{i}$ is a preconnection link, and so is $D_{j}$, where $D_{j}=D_{i+1}$ or $D_{j}=D_{i-1}$, and there is a continuum $K$ in $D_{i} \cup D_{j}$ which does not contain two disjoint preconnections, but which (1) intersects both $L_{i}$ and $R_{j}$ if $j=i+1$, or (2) intersects both $L_{j}$ and $R_{i}$ if $j=i-1$, then we say that $D_{i}$ is an adjoining preconnection link, and that $D_{i}$ adjoins $D_{j}$. Suppose then that $D_{i}$ and $D_{i+1}$ are adjoining preconnection links. Then there is an irreducible continuum $\widetilde{K}$ in $D_{i} \cup D_{i+1}$ which intersects both $L_{i}$ and $R_{i+1}$, but does not contain two disjoint preconnections. There is an irreducible subcontinuum $\Lambda_{i}$ of $\widetilde{K}$ in $D_{i}$ which intersects both $L_{i}$ and $R_{i+1}$. Then $\Lambda_{i} \cap \Lambda_{i+1} \neq \varnothing$, for otherwise $\widetilde{K}$ does contain two disjoint preconnections. Since $\widetilde{K}$ is irreducible between $L_{i}$ and $R_{i+1}, \widetilde{K}=\Lambda_{i} \cup \Lambda_{i+1}$.

4. Suppose that $\left\{D_{i}, D_{i+1}, \ldots, D_{i+m}\right\}$ is a subchain of $\underline{D}$ each link of which is an adjoining preconnection link. Then for each $i \leq j<j+1 \leq i+$ $m$, there is a continuum $K_{j}^{\prime}$ in $D_{j} \cup D_{j+1}$ which intersects both $L_{j}$ and $R_{j+1}$, but does not contain two disjoint preconnections. Then there is an irreducible subcontinuum $\Lambda_{j, j}$ of $K_{j}^{\prime}$ in $D_{j}$ which intersects both $L_{j}$ and $R_{j}$, and there is an irreducible subcontinuum $\Lambda_{j, j+1}$ in $D_{j+1}$ which intersects both $L_{j+1}$ and $R_{j+1}$. Then $\Lambda_{j, j} \cap \Lambda_{j, j+1} \neq \varnothing$, for otherwise $K_{j}^{\prime}$ does contain two disjoint preconnections, and $\Lambda_{j, u} \cup \Lambda_{j, j+1}$ is irreducible between $L_{j}$ and $R_{j+1}$. Since each $D_{j}^{\circ}$ is connected and locally connected, we can choose $K_{\underline{D}, j}$ to be a continuum irreducible between $L_{j}$ and $R_{j+1}$ such that $K_{\underline{D}, j} \cap L_{j}=$ $\Lambda_{j, j} \cap L_{j}, K_{\underline{D}, j} \cap R_{j}=\Lambda_{j, j} \cap R_{j}$, and $K_{\underline{D}, j} \cup K_{\underline{D}, j+1}$ does not contain two disjoint preconnections, and we can do this so that $\bigcup_{j=i}^{i+m} K_{\underline{D}, j}$ is a continuum irreducible between $L_{i}$ and $R_{m+i}$.

5 . If for some $i, D_{i}$ is a preconnection link which is not an adjoining preconnection link, then either no link that intersects $D_{i}$ is a preconnection link, or 
$D_{i+1}$ is a preconnection link, but no continuum $K$ in $D_{i} \cup D_{i+1}$ which intersects both $L_{i}$ and $R_{i+1}$ fails to contain two disjoint preconnections or $D_{i-1}$ is a preconnection link, but no continuum $K$ in $D_{i} \cup D_{i-1}$ which intersects both $L_{i-1}$ and $R_{i}$ fails to contain two disjoint preconnections. There is an irreducible continuum $K_{\underline{D}, i}$ in $D_{i}$ which intersects both $L_{i}$ and $R_{i}$. Further, since each $D_{i}^{\circ}$ is connected and locally connected, we can choose each $K_{\underline{D}, i}$ so that if $D_{j}$ is also a preconnection link and $D_{j}$ is adjacent to $D_{i}$, then $K_{\underline{D}, i} \cap K_{\underline{D}, j} \neq \varnothing$.

6. If $D_{i}$ is not a preconnection link, choose $K_{\underline{D}, i}$ to be an irreducible continuum from $L_{i}$ to $R_{i}$ contained in $D_{i}$ which is not a preconnection. (This can happen in one of two ways. Either for $j=0$ or $j=1$ both $L_{i}$ and $R_{i}$ are contained in $f^{-1}\left(e n d_{j}\right)$, or $D_{i} \nsubseteq f^{-1}(Q)$.) Again, since each $D_{i}^{\circ}$ is connected and locally connected, we can choose each $K_{\underline{D}, i}$ so that if $D_{j}$ is adjacent to $D_{i}$, then $K_{\underline{D}, i} \cap K_{\underline{D}, j} \neq \varnothing$.

Thus, $K_{\underline{D}}$ is a connection in $\bigcup \underline{D}$ which has the property that no connection $K^{\prime}$ has fewer mutually disjoint preconnections than does $K_{\underline{D}}$. Since each preconnection of $K_{\underline{D}}$ is contained in a link of $\underline{D}$, but no link of $\underline{D}$ contains more than one preconnection, it follows from Lemma 11 that the crossing number $M_{\underline{D}}$ for $\bigcup \underline{D}$ must be greater than or equal to $M$, and thus $\bigcup \underline{D}$ contains at least $M$ preconnection links such that the continua $K_{\underline{D}, i}$ corresponding to the $M_{\underline{D}}$ links in this set are mutually disjoint. More precisely, suppose that $\underline{D}^{\prime}=\left\{D_{\beta_{1}}, D_{\beta_{2}}, \ldots, D_{\beta_{N^{\prime \prime}}}\right\}$ denotes a maximal subcollection of preconnection links of $\underline{D}$ having the property that $\left\{K_{\underline{D}, \beta_{i}}: D_{\beta_{i}} \in \underline{D}^{\prime}\right\}$ is a collection of $M_{\underline{D}}$ disjoint continua. If $\underline{D}$ contains at least one subchain of at least 4 links consisting entirely of adjoining preconnection links, there may be more than one such set $\underline{D}^{\prime}$ for $\underline{D}$. However, this lack of uniqueness occurs only when $\underline{D}$ contains such a subchain (1) containing an even number greater than 2 links, and (2) this subchain is not properly contained in another subchain of preconnection links in $\underline{D}$. In this case, we choose as members of $\underline{D}^{\prime}$ the first, third, fifth, etc., members of the subchain of adjoining links rather than the second, fourth, etc., links.

Because distinct tiling chains can share links, though some of the preconnection links in the sets $\underline{D}^{\prime}$ may not "count": If $\underline{D}=\left\{D_{0}, D_{1}, \ldots, D_{N^{\prime}}\right\}$ and $\underline{E}=$ $\left\{E_{0}, E_{1}, \ldots, E_{N^{\prime \prime}}\right\}$ are irreducible end $d_{0}-e n d_{1} \mathcal{C}_{C o m}$ tiling chains, $D_{i}=E_{j}$, and no link of $\left\{D_{0}, D_{1}, \ldots, D_{i-1}\right\}$ is a link of $\left\{E_{j+1}, \ldots, E_{N^{\prime \prime}}\right\}$, then $\left\{D_{0}, D_{1}, \ldots, D_{k-1}\right.$, $\left.D_{i}, E_{j+1}, \ldots, E_{N^{\prime \prime}}\right\}$ is also an irreducible $e n d_{0}-e n d_{1} \mathcal{C}_{C o m}$ tiling chain. Thus if $\underline{D}=$ $\left\{D_{0}, D_{1}, \ldots, D_{N^{\prime}}\right\}$ is an irreducible $e n d_{0}-e n d_{1} \mathcal{C}_{C o m}$ tiling chain, we define $D_{\alpha(D)_{1}}$ to be the last link of $\underline{D}$ which is the first preconnection link of some irreducible end $_{0}$-end $d_{1} \mathcal{C}_{C o m}$ tiling chain $E$. (Since $\underline{D}$ has a first preconnection link, $\underline{D}$ has only finitely many preconnection links, and there are only finitely many irreducible end $d_{0}$-end $d_{1} \mathcal{C}_{C o m}$ tiling chains, $D_{\alpha(D)_{1}}$ exists.) We say that $D_{\alpha(D)_{1}}$ is the first essential preconnection link of $\underline{D}$. Next, we define $D_{\alpha(D)_{2}}$ to be the last link of $\underline{D}$ after $D_{\alpha(D)_{1}}$ which is the first nonadjoining preconnection link following the first essential preconnection link $E_{\alpha(E)_{1}}$ in some irreducible end $_{0}$-end $d_{1} \mathcal{C}_{C o m}$ tiling chain $\underline{E}$. Again, $D_{\alpha(D)_{2}}$ must exist (because otherwise we violate our basic assumption that each connection contains at least $M$ mutually disjoint preconnections), and we call it the second essential preconnection link of $\underline{D}$. This finite process continues until we choose the $M$ th essential preconnection link of $\underline{D}, D_{\alpha(D)_{M}}$ to be the last 
preconnection link of $\underline{D}$ after the link $D_{\alpha(D)_{M-1}}$ which is the first nonadjoining preconnection link after the link $E_{\alpha(D)_{M-1}}$ in some irreducible $e n d_{0}-e n d_{1} \mathcal{C}_{C o m}$ tiling chain $E$.

Then, for $1 \leq i \leq M, S_{C o m, i}=\bigcup\left\{D_{\alpha(D)_{i}}: D\right.$ is an irreducible end $_{0}-$ end $_{1} \mathcal{C}_{C o m}$ tiling chain $\}$. Finally, define for $1 \leq i \leq M, S_{i}=\bigcup\left\{S_{C o m, i}:\right.$ Com is a component of $Q\}$. Checking that this collection has the properties claimed is straightforward.

Outline of the proof of the Chaos Lemma. Suppose that each member $i_{*}=$ $\left(i_{j}\right)_{j=0}^{\infty}$ is in $\{1, \ldots, M\}$. Let $K_{*}^{\infty}=\left\{i_{*}: i_{*}\right.$ is a sequence of which each member is in $\{1, \ldots, M\}\}$, and for each $n \geq 0$, let $K_{*}^{n+1}=\left\{\left(i_{0}, i_{1}, i_{2}, \ldots, i_{n}\right)\right.$ : $\left(i_{0}, i_{1}, i_{2}, \ldots, i_{n}\right)$ is an $n+1$-member finite sequence of which each member is in $\{1, \ldots, M\}\}$. Define inductively $S_{\left(i_{0}, i_{1}\right)}=S_{i_{0}} \cap f^{-1}\left(S_{i_{1}}\right), S_{\left(i_{0}, i_{1}, i_{2}\right)}=S_{\left(i_{0}, i_{1}\right)} \cap$ $f^{-2}\left(S_{i_{2}}\right), \ldots, S_{\left(i_{0}, i_{1}, i_{2}, \ldots, i_{n}\right)}=S_{\left(i_{0}, i_{1}, \ldots, i_{n-1}\right)} \cap f^{-n}\left(S_{i_{n}}\right), \ldots$ It can be proved that $S_{i_{*}}=\bigcap_{j=0}^{\infty} S_{\left(i_{0}, i_{1}, i_{2}, \ldots, i_{n}\right)} \neq \varnothing$. Next, let $P=\bigcup_{i_{*} \in K^{\infty}} S_{i_{*}}$. Then $P$ is a compact subset of $Q$, and it can be written as $P=\bigcup_{i_{*} \in K_{*}^{\infty}}\left(\bigcap_{n=0}^{\infty} S_{\left(i_{0}, i_{1}, i_{2}, \ldots, i_{n}\right)}\right)=$ $\bigcap_{n=0}^{\infty}\left(\bigcup_{\left(i_{0}, i_{1}, i_{2}, \ldots, i_{n}\right) \in K_{*}^{n+1}} S_{\left(i_{0}, i_{1}, i_{2}, \ldots, i_{n}\right)}\right)$. Now $f(P) \subset P$. To get an invariant set, define $Y=\bigcap_{m=0}^{\infty} f^{m}(P)$ (i.e., $Y$ is a closed subset of $P$ such that $f(Y)=Y$ ). Since for each $i_{*}, Y_{i_{*}}=S_{i_{*}} \cap Y \neq \varnothing$ and $Y=\bigcup_{i_{*} \in K_{*}^{\infty}} Y_{i_{*}}$, there is a natural oneto-one correspondence between $Y$ and $K_{*}^{\infty}$ defined by $\alpha\left(Y_{i_{*}}\right)=i_{*}$. Let $g=f \mid Y$. Then $g: Y \rightarrow Y$ is continuous, $\alpha: Y \rightarrow K_{*}^{\infty}$, and it is not difficult to show that $\sigma \circ \alpha=\alpha \circ g$, where $\sigma$ denotes the one-sided shift on $M$ symbols.

The main theorem follows immediately from Lemma 12 and the Chaos Lemma.

\section{RElaxing the Hypotheses}

We do not need the strength of all the horseshoe hypotheses $\boldsymbol{\Omega}$, and we give lemmas below that enable the application of our results in some cases in which the ambient space $X$ is not locally compact. (This might occur, for example, when partial differential equations are studied.) Also, the last lemma shows that an extremely careful choice of $Q$ is not necessary, as long as it is precompact; in particular, it need not be the case that all components of $Q$ are connections. Any components that are not connections play no role in the choice of the symbol sets $\mathcal{S}$, but they do not cause problems either.

Suppose that $Y$ is a space, $Q$ is a closed, locally connected subset of $X$, and $f: X \rightarrow X$ is continuous. If $e n d_{0}$ and $e n d_{1}$ are disjoint closed subsets of $Q$, then we say that a closed, connected subset $K$ of $Q$ which intersects both end $d_{0}$ and $e n d_{1}$ is a end $d_{0}-e n d_{1}$ connection, or just a connection, if no confusion results. If $K^{\prime}$ is a closed connected subset of $Q \cap f^{-1}(Q)$ that intersects both $f^{-1}\left(e n d_{0}\right)$ and $f^{-1}\left(e n d_{1}\right)$, then $K^{\prime}$ is an end $d_{0}-e n d_{1}$ preconnection, or just a preconnection. We sometimes write $e n d(0)$ and $e n d(1)$ for $e n d_{0}$ and $e n d_{1}$. If $Q$ contains no $e n d_{0}-e n d_{1}$ connection or if each end $d_{0}-e n d_{1}$ connection contains no preconnection, then the crossing number of $f$ on $Q$ is 0 . If there is an end $d_{0}-e n d_{1}$ connection in $Q$ and each end $d_{0}-e n d_{1}$ connection contains at least one preconnection, then the crossing number of $f$ on $Q$ is the largest positive integer such that each connection $K$ in $Q$ contains at least $M$ mutually disjoint $e n d_{0}-e n d_{1}$ preconnections.

In this section we make the following five assumption:

$\boldsymbol{\Omega}_{X}^{\prime}: X$ is a separable metric space.

$\Omega_{Q}^{\prime}: Q \subset X$ is locally connected and closed. 
$\boldsymbol{\Omega}_{f}^{\prime}$ : The map $f: Q \rightarrow X$ is continuous, and $\overline{f(Q)}$ is a compact (i.e., $Q$ is precompact).

$\boldsymbol{\Omega}_{E}^{\prime}$ : The sets $e n d_{0} \subset Q$ and $e n d_{1} \subset Q$ are disjoint and compact, and at least one component of $Q$ intersects both $e n d_{0}$ and $e n d_{1}$.

$\boldsymbol{\Omega}_{M}^{\prime}: Q$ has a crossing number (defined below) $M \geq 2$.

We refer to the above five hypotheses $\boldsymbol{\Omega}_{X}^{\prime}, \boldsymbol{\Omega}_{Q}^{\prime}, \boldsymbol{\Omega}_{f}^{\prime}, \boldsymbol{\Omega}_{E}^{\prime}$, and $\boldsymbol{\Omega}_{M}^{\prime}$ collectively as the weak horseshoe hypotheses $\boldsymbol{\Omega}^{\prime}$. A connection $\Gamma$ is a closed connected subset of $Q$ that intersects both end $d_{0}$ and $e n d_{1}$. Note that by hypothesis $\boldsymbol{\Omega}_{E}^{\prime}$, at least one component of $Q$ is a connection. An example of a connection is a path in $Q$ intersecting both end $d_{0}$ and $e n d_{1}$. A preconnection $\gamma$ is a closed connected subset of $Q$ for which $f(\gamma)$ is a connection. We define the crossing number $M$ to be the largest number such that every connection contains at least $M$ mutually disjoint preconnections.

The last lemmas below argue that $Q$ contains a compact subset $Q^{\prime}$, all of whose connected components are connections, and furthermore that $Q^{\prime}$ has a crossing number at least as big as the crossing number $M$ of $Q$. When we later construct a compact invariant set in $Q^{\prime}$ that factors over an $M$-shift, the same will automatically be true of $Q$. Hence these two lemmas argue that without loss of generality, we can assume that $Q$ is compact and all its connected components are connections, since the weak horseshoe hypotheses $\boldsymbol{\Omega}^{\prime}$ on $Q$ with crossing number $M$ imply the horseshoe hypotheses $\boldsymbol{\Omega}$ on $Q^{\prime}$ with crossing number $M^{\prime} \geq M$.

Lemma 13. Suppose that $f: X \rightarrow X$ is continuous, and $f$ satisfies the weak horseshoe hypotheses $\boldsymbol{\Omega}^{\prime}$ on $Q$ with crossing number $M$ relative to ends end $d_{0}$ and end $d_{1}$. If $Q^{\prime}=Q \cap \overline{f(Q)}$, then $f$ satisfies $\boldsymbol{\Omega}$ on the compact set $Q^{\prime}$ with crossing number $M^{\prime} \geq M$ relative to ends end $d_{0}^{\prime}=e n d_{0} \cap \overline{f(Q)}$ and end $d_{1}^{\prime}=e n d_{1} \cap \overline{f(Q)}$.

Proof. Since $\overline{f(Q)}$ is compact, and $f$ satisfies $\boldsymbol{\Omega}^{\prime}$ on the closed set $Q$, there is some connection $K$ in $Q$. If $K$ is a connection in $Q$, the collection $\mathcal{K}_{p}=\left\{K_{\alpha}: K_{\alpha}\right.$ is a closed, connected subset of $K \cap f^{-1}(Q)$ that intersects both $f^{-1}\left(\right.$ end $\left._{0}\right)$ and $\left.f^{-1}\left(e n d_{1}\right)\right\}=\left\{K_{\alpha}: K_{\alpha}\right.$ is a preconnection in $\left.K\right\}$ has at least $M$ mutually disjoint members. Let $Q^{\prime}=Q \cap \overline{f(Q)}$. Then $Q^{\prime}$ is compact, and $Q^{\prime}$ contains a connection that is also a continuum. (For example, if $K_{\alpha}$ is a preconnection in the connection $K$ in $Q$, then $\overline{f\left(K_{\alpha}\right)}$ is a compact connection in $Q \cap \overline{f(Q)}$.). Furthermore, for each connection $K^{\prime}$ in $Q^{\prime}, \mathcal{K}_{p}^{\prime}=\left\{K_{\alpha}^{\prime}: K_{\alpha}^{\prime}\right.$ is a preconnection in $\left.K^{\prime}\right\}$ has at least $M$ mutually disjoint members. Thus, $Q^{\prime}$ is a compact subset of $Q$ on which $f$ satisfies $\boldsymbol{\Omega}$ relative to ends end $d_{0}^{\prime}=e n d_{0} \cap Q^{\prime}$ and end $d_{1}^{\prime}=e n d_{1} \cap Q^{\prime}$.

Lemma 14. Suppose that $f: X \rightarrow X$ is continuous, and $f$ satisfies the horseshoe hypotheses $\boldsymbol{\Omega}$ on the compact set $Q$ with crossing number $M$ relative to ends end 0 and end . Then $^{\prime}=\bigcup\{K: K$ is a connection in $Q$ and $K$ is a connected component of $Q\}$ is a compact subset of $Q$ and $f$ satisfies $\boldsymbol{\Omega}$ on $Q^{\prime}$ with crossing number $M^{\prime} \geq M$ relative to ends end $d_{0}^{\prime}=$ end $_{0} \cap Q^{\prime}$ and end $d_{1}^{\prime}=$ end $_{1} \cap Q^{\prime}$.

Proof. This is straightforward, and the proof is therefore omitted.

With Lemmas 13 and 14 we have reduced our problem to one of working with a compact set $Q$ of which each component is a connection. Thus, $f$ satisfies the horseshoe hypotheses $\Omega$ on a closed subset $Q^{\prime}$ of $Q$ with crossing number $M^{\prime} \geq M$ as long as $Q$ is precompact and contains at least one connection. Thus, we get the following slightly more general result: 
Theorem 15. More general version of the main result. Assume the weak horseshoe hypotheses $\boldsymbol{\Omega}^{\prime}$ on the set $Q$. Then there is a closed invariant set $Q_{I} \subset Q$ for which $f \mid Q_{I}$ is semiconjugate to a one-sided $M$-shift. (If $f$ is a homeomorphism, then $f \mid Q_{I}$ is also semiconjugate to a two-sided $M$-shift).

\section{REFERENCES}

[BW] K. Burns and H. Weiss, A geometric criterion for positive topological entropy, Commun. Math. Phys. 172 (1995), 95-118. MR 96e:58120

[CKM] M. Carbinatto, J. Kwapisz, and K. Mischaikow, Horseshoes and the Conley index spectrum, Ergodic Theory Dynam. Systems 20 (2000), 365-377. CMP 2000:12

[CM] M. Carbinatto and K. Mischaikow, Horseshoes and the Conley index spectrum II. The theorem is sharp, Discrete Contin. Dynam. Systems 5 (1999), 599-616. MR 2000i:37009

[KKY] J. Kennedy, S. Koçak, and J. Yorke, A Chaos Lemma, to appear, Amer. Math. Monthly.

[KSYG] J. Kennedy, M. Sanjuan, J. Yorke, and C. Grebogi, The topology of fluid flow past a sequence of cylinders, Topology and its Applications 94 (1999), 207-242. CMP 99:14

$[\mathrm{Ku}$ C. Kuratowski, Topology II, Academic Press, New York, 1968. MR 41:4467

[MM] K. Mischaikow and M. Mrozek, Isolating Neighborhoods and Chaos, Japan Journal of Industrial and Applied Mathematics 12 (1995), 205-236. MR 96e:58104

[S] A. Szymczak, The Conley index and symbolic dynamics, Topology 35 (1996), 287-299. MR 97b:58054

[SKGY] M. Sanjuan, J. Kennedy, C. Grebogi, and J. Yorke, Indecomposable continua in dynamical systems with noise: fluid flow dynamics past a spatial array of cylinders, Chaos $\mathbf{7}$ (1997), 125-138. MR 98a:76027

Department of Mathematical Sciences, University of Delaware, Newark, Delaware 19716

E-mail address: jkennedy@math.udel.edu

Institute for Physical Science and Technology, University of Maryland, College Park, Maryland 20742

E-mail address: yorke@ipst.umd.edu 